\title{
Possible interpretation of the newly observed $\Omega(2012)$ state
}

\author{
Li-Ye Xiao ${ }^{1,2, *}$ and Xian-Hui Zhong ${ }^{3,4, \dagger}$ \\ ${ }^{1}$ School of Physics and State Key Laboratory of Nuclear Physics and Technology, \\ Peking University, Beijing 100871, China \\ ${ }^{2}$ Center of High Energy Physics, Peking University, Beijing 100871, China \\ ${ }^{3}$ Department of Physics, Hunan Normal University, and Key Laboratory of Low-Dimensional Quantum \\ Structures and Quantum Control of Ministry of Education, Changsha 410081, China \\ ${ }^{4}$ Synergetic Innovation Center for Quantum Effects and Applications (SICQEA), \\ Hunan Normal University, Changsha 410081, China
}

(Received 5 June 2018; published 3 August 2018)

\begin{abstract}
Inspired by the newly observed $\Omega(2012)$ state at Belle II, we investigate the two-body strong decays of $\Omega$ baryons up to $N=2$ shell within the chiral quark model. Our results indicate that (i) the newly observed $\Omega(2012)$ state could be assigned to the spin-parity $J^{P}=3 / 2^{-}$state $\left|70,{ }^{2} 10,1,1, \frac{3}{2}-\right\rangle$ and the experimental data can be reasonably described. However, the spin-parity $J^{P}=1 / 2^{-}$state $\left|70,{ }^{2} 10,1,1, \frac{1}{2}^{-}\right\rangle$ and spin-parity $J^{P}=3 / 2^{+}$state $\left|56,{ }^{4} 10,2,0, \frac{3}{2}{ }^{+}\right\rangle$cannot be completely excluded. (ii) The $D$-wave states in the $N=2$ shell are most likely to be narrow states with a width of dozens of $\mathrm{MeV}$ and have a good potential to be observed in the $\Xi K$ and/or $\Xi(1530) K$ channels in future experiments. The $\Omega(2250)$ resonance listed in PDG may be a good candidate for the $J^{P}=5 / 2^{+} 1 D$ wave state $\left|56,{ }^{4} 10,2,2,5 / 2^{+}\right\rangle$.
\end{abstract}

DOI: 10.1103/PhysRevD.98.034004

\section{INTRODUCTION}

Searching for the missing baryon resonances and understanding the baryon spectrum are important topics in hadron physics. In recent years, due to the limitations of experimental conditions, our knowledge about the $\Omega$ spectrum has remained scarce. There are only a few data on the $\Omega$ resonances. In the review of the Particle Data Group (PDG) [1], only four $\Omega$ baryon states are listed: $\Omega(1672), \Omega(2250), \Omega(2380)$, and $\Omega(2470)$. Except for the ground state $\Omega(1672)$ being well established with four-star ratings, the nature of the other three excited states is still rather uncertain with three- or two-star ratings. Fortunately, the Belle II experiments offer a great opportunity for our study of the $\Omega$ spectrum.

Very recently, Belle II Collaboration reported a new excited hyperon, an $\Omega^{*-}$ candidate (denoted by $\Omega(2012)$ here), in the $\Xi^{0} K^{-}$and $\Xi^{-} K^{0}$ mass distributions [2]. Its mass and decay width are measured to be

$$
M=2012.4 \pm 0.7 \text { (stat) } \pm 0.6(\text { syst }) \mathrm{MeV}
$$

\footnotetext{
*lyxiao@pku.edu.cn

tzhongxh@hunnu.edu.cn
}

Published by the American Physical Society under the terms of the Creative Commons Attribution 4.0 International license. Further distribution of this work must maintain attribution to the author(s) and the published article's title, journal citation, and DOI. Funded by SCOAP ${ }^{3}$. and

$$
\Gamma=6.4_{-2.0}^{+2.5}(\text { stat }) \pm 0.6(\text { syst }) \mathrm{MeV},
$$

respectively. In various models and methods, such as the Skyrme model [3], quark model [4-13], lattice gauge theory [14,15] and so on [16-21], the masses of the first orbital excitations of $\Omega(1672)$ are predicted to be $\sim 2.0 \mathrm{GeV}$. Thus, the newly observed $\Omega(2012)$ may be a good candidate for the first orbital $(1 P)$ excitations of $\Omega(1672)$. In Ref. [8], the mass of $2 S$ state (the first radially excitation of $\Omega(1672)$ ) with $J^{P}=3 / 2^{+}$was predicted to be $2065 \mathrm{MeV}$, which is also close to the mass of $\Omega(2012)$. To further determine the spin-parity quantum numbers and inner structure of $\Omega(2012)$, besides its mass one should study its other properties, such as magnetic moments, radiative and strong decays properties, as well. In the early literature, limited discussions exist on the magnetic moments [22], radiative decays [23] of the $\Omega$ resonances. In present work we will attempt to understand the inner structure of this newly observed state $\Omega(2012)$ by analyzing the strong decay properties of its possible candidates within a chiral quark model, so that our theoretical widths can be compared with the measured width directly.

The chiral quark model [24] is developed and successfully used to study the Okubo-Zweig-Iizuka (OZI) allowed two-body strong decays of the heavy-light mesons [25-28] and baryons [29-36]. In this framework, the spatial wave functions of heavy baryons are described by harmonic 
TABLE I. The theoretical masses (MeV) and spin-flavor-space wavefunctions of baryons, denoted by $\left|\mathbf{N}_{6},{ }^{2 S+1} \mathbf{N}_{3}, N, L, J^{P}\right\rangle[31]$. The Clebsch-Gordan series for the spin and angular-momentum addition $\left|J, J_{z}\right\rangle=\sum_{L_{z}+S_{z}=J_{z}}\left\langle L L_{z}, S S_{z} \mid J J_{z}\right\rangle \Psi_{N L L_{z}}^{\sigma} \chi_{S_{z}}$ has been omitted.

\begin{tabular}{|c|c|c|c|c|c|c|}
\hline \multirow[b]{2}{*}{ State } & \multirow[b]{2}{*}{ Wave function } & \multirow[b]{2}{*}{ Experiment [1] } & \multicolumn{4}{|c|}{ Theory } \\
\hline & & & [5] & [4] & [3] & [8] \\
\hline$\left|56,{ }^{4} 10,0,0, \frac{3}{2}+\right\rangle$ & $\left|56,{ }^{4} 10\right\rangle \Psi_{000}^{s}$ & 1672.45 & 1678 & 1635 & 1694 & 1675 \\
\hline$\left|70,{ }^{2} 10,1,1, \frac{1}{2}^{-}\right\rangle$ & $\left|70,{ }^{2} 10\right\rangle^{\rho} \Psi_{11 L_{z}}^{\rho}+\left|70,{ }^{2} 10\right\rangle^{\lambda} \Psi_{11 L_{z}}^{\lambda}$ & $\cdots$ & 1941 & 1950 & 1837 & 2020 \\
\hline$\left|70,{ }^{2} 10,1,1, \frac{3}{2}-\right\rangle$ & $\left|70,{ }^{2} 10\right\rangle^{\rho} \Psi_{11 L_{z}}^{\rho}+\left|70,{ }^{2} 10\right\rangle^{\lambda} \Psi_{11 L_{z}}^{\lambda}$ & $\ldots$ & 2038 & 2000 & 1978 & 2020 \\
\hline$\left|70,{ }^{2} 10,2,0, \frac{1}{2}^{+}\right\rangle$ & $\left|70,{ }^{2} 10\right\rangle^{\rho} \Psi_{200}^{\rho}+\left|70,{ }^{2} 10\right\rangle^{\lambda} \Psi_{200}^{\lambda}$ & $\ldots$ & 2301 & 2220 & 2140 & 2190 \\
\hline$\left|56,{ }^{4} 10,2,2, \frac{1}{2}+\right\rangle$ & $\left|56,{ }^{4} 10\right\rangle \Psi_{22 L_{z}}^{s}$ & $\ldots$ & & 2255 & & 2210 \\
\hline$\left|56,{ }^{4} 10,2,0, \frac{3}{2}^{+}\right\rangle$ & $\left|56,{ }^{4} 10\right\rangle \Psi_{200}^{s}$ & $\ldots$ & $2173 / 2304$ & 2165 & 2282 & 2065 \\
\hline$\left|56,{ }^{4} 10,2,2, \frac{3}{2}+\right\rangle$ & $\left|56,{ }^{4} 10\right\rangle \Psi_{22 L_{z}}^{s}$ & $\ldots$ & & 2280 & & 2215 \\
\hline$\left|70,{ }^{2} 10,2,2, \frac{3}{2}^{+}\right\rangle$ & $\left|70,{ }^{2} 10\right\rangle^{\rho} \Psi_{22 L_{z}}^{\rho}+\left|70,{ }^{2} 10\right\rangle^{\lambda} \Psi_{22 L_{z}}^{\lambda}$ & $\cdots$ & & 2345 & & 2265 \\
\hline$\left|56,{ }^{4} 10,2,2, \frac{5}{2}^{+}\right\rangle$ & $\left|56,{ }^{4} 10\right\rangle \Psi_{22 L_{z}}^{s}$ & $\cdots$ & 2401 & 2280 & $\cdots$ & 2225 \\
\hline$\left|70,{ }^{2} 10,2,2, \frac{5}{2}^{+}\right\rangle$ & $\left|70,{ }^{2} 10\right\rangle^{\rho} \Psi_{22 L_{z}}^{\rho}+\left|70,{ }^{2} 10\right\rangle^{\lambda} \Psi_{22 L_{z}}^{\lambda}$ & $\cdots$ & & 2345 & $\cdots$ & 2265 \\
\hline$\left|56,{ }^{4} 10,2,2, \frac{7}{2}^{+}\right\rangle$ & $\left|56,{ }^{4} 10\right\rangle \Psi_{22 L_{z}}^{s}$ & $\cdots$ & 2332 & 2295 & $\cdots$ & 2210 \\
\hline
\end{tabular}

oscillators, and an effective chiral Lagrangian is then introduced to account for the quark-meson coupling at the baryon-meson interaction vertex. The light pseudoscalar mesons, i.e., $\pi, K$, and $\eta$, are treated as Goldstone bosons. Since the quark-meson coupling is invariant under the chiral transformation, some of the low-energy properties of QCD are retained [24,37-39]. Within the chiral quark model, the OZI allowed two-body strong decays of $\Omega$ baryons up to $N=2$ shell are analyzed in present work. The quark model classification for the $\Omega$ baryons and their theoretical masses are listed in Table I. According to our calculations, we obtain that (i) the newly observed $\Omega(2012)$ resonance could be assigned to the spin-parity $J^{P}=3 / 2^{-}$state $\left|70,{ }^{2} 10,1,1, \frac{3}{2}-\right\rangle$ and the experimental data can be reasonably described. However, the spin-parity $J^{P}=1 / 2^{-}$state $\left|70,{ }^{2} 10,1,1, \frac{1}{2}\right\rangle$ and spin-parity $J^{P}=3 / 2^{+}$state $\left|56,{ }^{4} 10,2,0, \frac{3}{2}^{+}\right\rangle$cannot be completely excluded. (ii) The $D$-wave states in the $N=2$ shell are most likely to be narrow states with a width of dozens of $\mathrm{MeV}$ and have a good potential to be observed in their corresponding dominant decay channels. The $\Omega(2250)$ resonance listed in PDG may be a good candidate for the $J^{P}=5 / 2^{+} 1 D$ wave state $\left|56,{ }^{4} 10,2,2,5 / 2^{+}\right\rangle$.

This paper is organized as follows. In Sec. II, we give a brief introduction of the chiral quark model. We present our numerical results and discussions in Sec. III and summarize our results in Sec. IV.

\section{THE CHIRAL QUARK MODEL}

In the chiral quark model, the effective low-energy quark-pseudoscalar-meson coupling in the $\mathrm{SU}(3)$ flavor basis at tree level is given by [24]

$$
H_{m}=\sum_{j} \frac{1}{f_{m}} \bar{\psi}_{j} \gamma_{\mu}^{j} \gamma_{5}^{j} \psi_{j} \vec{\tau} \cdot \partial^{\mu} \vec{\phi}_{m},
$$

where $f_{m}$ stands for the pseudoscalar meson decay constant. $\psi_{j}$ corresponds to the $j$ th quark field in a baryon and $\phi_{m}$ denotes the pseudoscalar meson octet

$$
\phi_{m}=\left(\begin{array}{ccc}
\frac{1}{\sqrt{2}} \pi^{0}+\frac{1}{\sqrt{6}} \eta & \pi^{+} & K^{+} \\
\pi^{-} & -\frac{1}{\sqrt{2}} \pi^{0}+\frac{1}{\sqrt{6}} \eta & K^{0} \\
K^{-} & \bar{K}^{0} & -\sqrt{\frac{2}{3} \eta}
\end{array}\right) .
$$

To match the nonrelativistic harmonic oscillator spatial wave function $\Psi_{N L L_{z}}$ in the calculations, we adopt a nonrelativistic form of Eq. (3) and get [37-39]

$$
\begin{aligned}
H_{m}^{n r}= & \sum_{j}\left\{\frac{\omega_{m}}{E_{f}+M_{f}} \boldsymbol{\sigma}_{j} \cdot \mathbf{P}_{f}+\frac{\omega_{m}}{E_{i}+M_{i}} \boldsymbol{\sigma}_{j} \cdot \mathbf{P}_{i}-\boldsymbol{\sigma}_{j} \cdot \mathbf{q}\right. \\
& \left.+\frac{\omega_{m}}{2 \mu_{q}} \boldsymbol{\sigma}_{j} \cdot \mathbf{p}_{j}^{\prime}\right\} I_{j} \varphi_{m},
\end{aligned}
$$

where $\left(E_{i}, \mathbf{p}_{i}\right),\left(E_{f}, \mathbf{p}_{f}\right)$, and $\left(\omega_{m}, \mathbf{q}\right)$ stand for the energy and three-vector momentum of the initial baryon, final baryon, and meson, respectively. $\sigma_{j}$ is the Pauli spin vector on the $j$ th quark, and $\mu_{q}$ is a reduced mass expressed as $1 / \mu_{q}=1 / m_{j}+1 / m_{j}^{\prime} \cdot \mathbf{p}_{j}^{\prime}=\mathbf{p}_{j}-\left(m_{j} / M\right) \mathbf{P}_{\mathrm{c} . \mathrm{m} .}$ is the internal momentum of the $j$ th quark in the baryon rest frame. $\varphi_{m}=e^{-i \mathbf{q} \cdot \mathbf{r}_{j}}$ and $e^{i \mathbf{q} \cdot \mathbf{r}_{j}}$ for emitting and absorbing a meson, respectively. The isospin operator $I_{j}$ associated with the pseudoscalar meson is given by 


$$
I_{j}= \begin{cases}a_{j}^{\dagger}(u) a_{j}(s) & \text { for } K^{-}, \\ a_{j}^{\dagger}(d) a_{j}(s) & \text { for } \bar{K}^{0}, \\ \frac{1}{\sqrt{2}}\left[a_{j}^{\dagger}(u) a_{j}(u)+a_{j}^{\dagger}(d) a_{j}(d)\right] \cos \theta & \\ -a_{j}^{\dagger}(s) a_{j}(s) \sin \theta & \text { for } \eta .\end{cases}
$$

Here, $a_{j}^{\dagger}(u, d, s)$ and $a_{j}(u, d, s)$ are the creation and annihilation operator for the $u, d, s$ quarks on $j$ th quark. $\theta$ is the mixing angle of the $\eta$ meson in the flavor basis [1].

For the decay processes, we select the initial-baryon-rest system in the calculations. Then, $\mathbf{p}_{i}=0$ and $\mathbf{p}_{f}=-\mathbf{q}$. The Eq. (5) can be further simplified and the partial decay amplitudes for $\mathcal{B} \rightarrow \mathcal{B}^{\prime} \mathbb{M}$ can be calculated by

$\mathcal{M}\left[\mathcal{B} \rightarrow \mathcal{B}^{\prime} \mathbb{M}\right]=3\left\langle\mathcal{B}^{\prime}\left|\left\{G \boldsymbol{\sigma}_{3} \cdot \mathbf{q}+h \boldsymbol{\sigma}_{3} \cdot \mathbf{p}_{3}^{\prime}\right\} I_{3} e^{-i \mathbf{q} \cdot \mathbf{r}_{3}}\right| \mathcal{B}\right\rangle$,

with

$$
h \equiv \frac{\omega_{m}}{2 \mu_{q}}, \quad G \equiv-\left(\frac{\omega_{m}}{E_{f}+M_{f}}+1\right),
$$

where $\mathcal{B}^{\prime}$ and $\mathcal{B}$ stand for the final and initial baryon wave functions listed in Table I.

With the derived decay amplitudes, the partial decay width for the emission of a light pseudoscalar meson is calculated by

$$
\Gamma=\left(\frac{\delta}{f_{m}}\right)^{2} \frac{\left(E_{f}+M_{f}\right)|\mathbf{q}|}{4 \pi M_{i}} \frac{1}{2 J_{i}+1} \sum_{J_{i z} J_{f z}}\left|\mathcal{M}_{J_{i z}, J_{f z}}\right|^{2},
$$

where $J_{i z}$ and $J_{f z}$ represent the third components of the total angular momenta of the initial and final baryons, respectively. $\delta$ is a global parameter accounting for the strength of the quark-meson couplings.

The relativistic effect should be significant when momentum $\mathbf{q}$ of final baryon increases [40]. To partly remedy the inadequacy of the nonrelativistic wave function as the momentum $\mathbf{q}$ increases, a commonly used Lorentz boost factor $\gamma_{f}$ is introduced into the decay amplitudes $[25,41-43]$

$$
\mathcal{M}(\mathbf{q}) \rightarrow \gamma_{f} \mathcal{M}\left(\gamma_{f} \mathbf{q}\right),
$$

where $\gamma_{f} \equiv M_{f} / E_{f}$. In most decays, the three momenta $\mathbf{q}$ carried by the final state mesons are relatively small, which means the nonrelativistic prescription is reasonable and corrections from the Lorentz boost are not drastic.

In this work, the standard quark model parameters are adopted. Namely, we set $m_{s}=450 \mathrm{MeV}$ for the constituent quark mass. The decay constants for $K$ and $\eta$ are taken as $f_{K}=f_{\eta}=160 \mathrm{MeV}$. The harmonic oscillator parameter $\alpha$ in the wave function $\Psi_{N L L_{z}}$ is adopted as $\alpha=400 \mathrm{MeV}$. The masses of the final mesons and baryons are taken from the PDG [1]. For the global parameter $\delta$, we fix its value the same as our previous study of the strong decays of $\Xi$ baryons [31], i.e., $\delta=0.576$.

\section{RESULTS AND ANALYSIS}

Inspired by the newly observed $\Omega^{*-}$ candidate by Belle II Collaboration [2], we carry out a systematic study of the strong decays of $\Omega$ baryons up to the $N=2$ shell with a chiral quark model. Since the predicted mass of the $\Omega^{-}$in the relativistic quark model [8] well agrees with the experimental measurement in PDG [1], we adopt the predicted masses of the $\Omega$ resonances from Ref. [8] (see Table I) in our calculation.

\section{A. $1 P$ wave states in the $N=1$ shell}

There are two $1 P$ wave states $\left|70,{ }^{2} 10,1,1,1 / 2^{-}\right\rangle$and $\left|70,{ }^{2} 10,1,1,3 / 2^{-}\right\rangle$according to the quark model classification (see Table I). Their spin-parity quantum numbers are $J^{P}=1 / 2^{-}$and $J^{P}=3 / 2^{-}$, respectively. It is seen that the predicted masses in various quark models for these two $1 P$ wave states are about $2000 \mathrm{MeV}$, which are close to the mass of the newly observed $\Omega^{*-}$ candidate [2]. As the possible assignments of the newly observed $\Omega(2012)$ state, it is crucial to study the decay properties of the two states.

Considering the $\Omega(2012)$ state as candidates for both the $J^{P}=1 / 2^{-}$and $J^{P}=3 / 2^{-}$states, we calculate their strong decay properties; our results are shown in Table II. As a candidate for the $J^{P}=3 / 2^{-}$state $\left|70,{ }^{2} 10,1,1,3 / 2^{-}\right\rangle$, the predicted width

$$
\Gamma_{\text {total }}^{\text {th }}[\Omega(2012)]=5.03 \mathrm{MeV},
$$

and branching fraction ratio

$$
\mathcal{R}=\frac{\mathcal{B}\left[\Omega(2012) \rightarrow \Xi^{0} K^{-}\right]}{\mathcal{B}\left[\Omega(2012) \rightarrow \Xi^{-} \bar{K}^{0}\right]} \simeq 1.10
$$

are highly consistent with the measured width $\Gamma^{\exp }=$ $6.4_{-2.0}^{+2.5} \pm 0.6 \mathrm{MeV}$ and ratio $\mathcal{R}^{\exp }=1.2 \pm 0.3$ of the newly observed $\Omega(2012)$ state.

It should be pointed out that if $\Omega(2012)$ indeed corresponds to the $\left|70,{ }^{2} 10,1,1,3 / 2^{-}\right\rangle$configuration, the $\Delta(1700)$ state with spin-parity $J^{P}=\frac{3}{2}-$ listed in PDG [1] is most likely to be a $\mathrm{SU}(3)$ partner of $\Omega(2012)$ [44]. As suggested in Ref. [45], to obtain a combined understanding

TABLE II. The predicted partial and total decay widths $(\mathrm{MeV})$ of the assignments $\left|70,{ }^{2} 10,1,1,1 / 2^{-}\right\rangle$and $\left|70,{ }^{2} 10,1,1,3 / 2^{-}\right\rangle$ with a mass of $M=2012 \mathrm{MeV}$.

\begin{tabular}{lccc}
\hline \hline States & $\Gamma\left[\Xi^{0} K^{-}\right]$ & $\Gamma\left[\Xi^{-} \bar{K}^{0}\right]$ & $\Gamma_{\text {total }}^{\text {th }}$ \\
\hline$\left|70,{ }^{2} 10,1,1,1 / 2^{-}\right\rangle$ & 7.50 & 7.81 & 15.3 \\
$\left|70,{ }^{2} 10,1,1,3 / 2^{-}\right\rangle$ & 2.64 & 2.39 & 5.03 \\
\hline \hline
\end{tabular}


TABLE III. Partial decay width $\Gamma_{i}(\mathrm{MeV})$, total decay width $\Gamma(\mathrm{MeV})$ and fraction $\Gamma_{i} / \Gamma$ of the resonance $\Delta(1700)$ compared with the data from the PDG [1]. The $\Delta(1700)$ state is considered as a candidate for $\left|70,{ }^{2} 10,1,1,3 / 2^{-}\right\rangle$with a mass of $M=1710 \mathrm{MeV}$.

\begin{tabular}{lccc}
\hline \hline Mode & $\Gamma_{i}$ & $\Gamma_{i} / \Gamma$ & $\Gamma_{i} / \Gamma($ Exp.) $[1]$ \\
\hline$N \pi$ & 8.97 & $12 \%$ & $10-20 \%$ \\
$\Delta(1232) \pi$ & 60.4 & $80 \%$ & $10-50 \%$ \\
$N(1520) \pi$ & 4.29 & $5.7 \%$ & $1-5 \%$ \\
$N(1535) \pi$ & 1.93 & $2.6 \%$ & $0.5-1.5 \%$ \\
$\Sigma K$ & 0.01 & $0.01 \%$ & $\ldots$ \\
$\Gamma_{\text {total }}^{\text {th }}$ & 76 & $\Gamma_{\text {total }}^{\exp }$ & $220-380[1]$ \\
\hline \hline
\end{tabular}

of these two $J^{P}=\frac{3}{2}^{-}$baryons, we study the strong decays of $\Delta(1700)$ as a assignment of $\left|70,{ }^{2} 10,1,1,3 / 2^{-}\right\rangle$as well. Our results are listed in Table III. From the table, it is found that the fractions $\Gamma_{i} / \Gamma$ predicted with our chiral quark model are in good agreement with the data from the PDG [1], although our predicted total width for $\Delta(1700)$,

$$
\Gamma_{\text {total }}^{\text {th }}[\Delta(1700)] \simeq 76 \mathrm{MeV},
$$

is about a factor of 3 smaller than the lower limit estimated by the PDG [1]. It should be emphasized that the measured width of $\Delta(1700)$ still has a large uncertainty. We also find that in Ref. [46] the authors extracted a narrow width of $\Gamma \simeq 119 \pm 70 \mathrm{MeV}$ for $\Delta(1700)$ from the $\pi N$ data using a unitary multichannel model, which is consistent with our prediction. Thus, assigning both $\Delta(1700)$ and $\Omega(2012)$ as the same configuration $\left|70,{ }^{2} 10,1,1,3 / 2^{-}\right\rangle$in the quark model, their decay properties can be reasonably understood.

Meanwhile, as a candidate for the $J^{P}=1 / 2^{-}$state $\left|70,{ }^{2} 10,1,1,1 / 2^{-}\right\rangle$, the predicted width for $\Omega(2012)$ is

$$
\Gamma_{\text {total }}^{\text {th }}[\Omega(2012)]=15.3 \mathrm{MeV},
$$

and the branching fraction ratio is predicted to be

$$
\mathcal{R}=\frac{\mathcal{B}\left[\Omega(2012) \rightarrow \Xi^{0} K^{-}\right]}{\mathcal{B}\left[\Omega(2012) \rightarrow \Xi^{-} \bar{K}^{0}\right]} \simeq 0.96
$$

The predicted total decay width of is about 2.5 times larger than the measured width of $\Omega(2012)$. Considering the model uncertainties, the possibility as the assignment of $J^{P}=1 / 2^{-}$state $\left|70,{ }^{2} 10,1,1,1 / 2^{-}\right\rangle$can't be excluded.

Considering the uncertainties of the predicted masses, we plot the variation of the decay properties of the two states as functions of the masses in Fig. 1. The total decay width of $\left|70,{ }^{2} 10,1,1,1 / 2^{-}\right\rangle$is about $\Gamma \sim(14-18) \mathrm{MeV}$ with the mass varied in the range of (1900-2100) MeV and insensitive to its mass within the considered range. For the state $\left|70,{ }^{2} 10,1,1,3 / 2^{-}\right\rangle$, if it lies below the threshold of $\Xi(1530) K$, its dominant decay mode is $\Xi K$ with a fairly
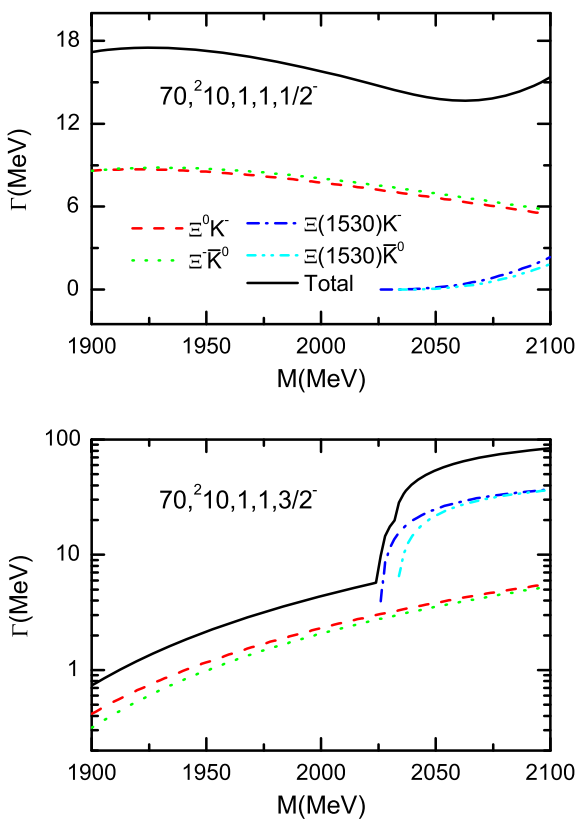

FIG. 1. The strong decay properties of the $1 P$-wave states in the $N=1$ shell.

narrow width $\Gamma<6 \mathrm{MeV}$. However, if its mass is above the threshold of $\Xi(1530) K$, it mainly decays into the $\Xi(1530) K$ channel and its total decay width may reach up to $\Gamma \sim 84 \mathrm{MeV}$ with the mass $M=2100 \mathrm{MeV}$.

\section{B. $2 S$ wave states in the $N=2$ shell}

In the quark model, there are two $2 S$ wave states with $J^{P}=1 / 2^{+}$and $J^{P}=3 / 2^{+}$, i.e., $\left|70,{ }^{2} 10,2,0,1 / 2^{+}\right\rangle$and $\left|56,{ }^{4} 10,2,0,3 / 2^{+}\right\rangle$. In Ref. [8], their masses were predicted to be about $2.19 \mathrm{GeV}$ and $2.06 \mathrm{GeV}$ [8], respectively. Using these predicted masses, we calculate their partial and total strong decay widths. Our results have been listed in Table IV. It is found that both $\left|70,{ }^{2} 10,2,0,1 / 2^{+}\right\rangle$and $\left|56,{ }^{4} 10,2,0,3 / 2^{+}\right\rangle$are most likely to be fairly narrow states with a width of $\Gamma \sim 2.4 \mathrm{MeV}$.

The dominant decay mode of $\left|70,{ }^{2} 10,2,0,1 / 2^{+}\right\rangle$ is $\Xi(1530) K$. While the dominant decay modes of $\left|56,{ }^{4} 10,2,0,3 / 2^{+}\right\rangle$are $\Xi K$ and $\Xi(1530) K$, and the predicted branching fraction ratio is

$$
\frac{\mathcal{B}\left[\left|56,{ }^{4} 10,2,0,3 / 2^{+}\right\rangle \rightarrow \Xi K\right]}{\mathcal{B}\left[\left|56,{ }^{4} 10,2,0,3 / 2^{+}\right\rangle \rightarrow \Xi(1530) K\right]} \simeq 1.57 .
$$

From the point of view of the mass and decay width, we can't excluded the first radially excited $\Omega$ state $\left|56,{ }^{4} 10,2,0,3 / 2^{+}\right\rangle$as a assignment of the newly observed $\Omega(2012)$ state.

In addition, we also plot the decay widths of $\left|70,{ }^{2} 10,2,0,1 / 2^{+}\right\rangle$and $\left|56,{ }^{4} 10,2,0,3 / 2^{+}\right\rangle$as functions of the masses in the range of $M=(2000-2300) \mathrm{MeV}$ in 
TABLE IV. The predicted partial and total decay widths of the $S$ - and $D$-wave states in the $N=2$ shell. $\Gamma_{\text {total }}^{\text {th }}$ stands for the total decay width and $\mathcal{B}$ represents the ratio of the branching fraction $\Gamma[\Xi K] / \Gamma[\Xi(1530) K]$. The unit of widths and masses is MeV.

\begin{tabular}{cccccccc}
\hline \hline & States & Mass $[8]$ & $\Gamma[\Xi K]$ & $\Gamma[\Xi(1530) K]$ & $\Gamma[\Omega \eta]$ & $\Gamma_{\text {total }}^{\text {th }}$ & $\mathcal{B}$ \\
\hline \multirow{2}{*}{$1 D$ wave } & $\left|70,{ }^{2} 10,2,0,1 / 2^{+}\right\rangle$ & 2190 & 0.01 & 2.39 & $\ldots$ & 2.40 & 0.00 \\
& $\left|56,{ }^{4} 10,2,0,3 / 2^{+}\right\rangle$ & 2065 & 1.48 & 0.94 & $\ldots$ & 2.42 & 1.57 \\
& $\left|56,{ }^{4} 10,2,2,1 / 2^{+}\right\rangle$ & 2210 & 48.8 & 4.09 & $\ldots$ & 52.9 & 11.9 \\
& $\left|56,{ }^{4} 10,2,2,3 / 2^{+}\right\rangle$ & 2215 & 24.4 & 14.0 & $\ldots$ & 38.4 & 1.74 \\
& $\left|56,{ }^{4} 10,2,2,5 / 2^{+}\right\rangle$ & 2225 & 3.19 & 20.0 & 0.11 & 23.3 & 0.16 \\
& $\left|56,{ }^{4} 10,2,2,7 / 2^{+}\right\rangle$ & 2210 & 13.0 & 1.16 & $\ldots$ & 14.2 & 11.2 \\
& $\left|70,{ }^{2} 10,2,2,3 / 2^{+}\right\rangle$ & 2265 & 3.82 & 10.8 & 1.56 & 16.2 & 0.35 \\
& $\left|70,{ }^{2} 10,2,2,5 / 2^{+}\right\rangle$ & 2265 & 1.30 & 9.56 & 0.81 & 11.7 & 0.14 \\
\hline \hline
\end{tabular}

Fig. 2. The variation curves between the decay widths and the masses of the two states can be obtained from the figure.

\section{C. $1 D$ wave states in the $N=2$ shell}

There are six $1 D$ wave states according to the quark model classification (see Table I). Their masses are estimated to be in the range of $2.2-2.3 \mathrm{GeV}$ in various quark models. With the predicted masses from Ref. [8], we further analyze the decay properties of the $1 D$ wave states in the $N=2$ shell, and collect their strong decay widths in Table IV. The predicted masses of the $1 D$ wave states certainly have a large uncertainty, which may bring uncertainties to our theoretical predictions. To investigate this effect, we plot the total and partial decay widths of these states as functions of the masses in the range of $M=$ (2100-2400) MeV in Fig. 2 as well.

It is found that the total decay widths of the $1 D$ wave states are not broad, they are about $\Gamma \simeq(3-70) \mathrm{MeV}$. The strong decays of both $\left|56,{ }^{4} 10,2,2,1 / 2^{+}\right\rangle$and $\left|56,{ }^{4} 10,2,2,7 / 2^{+}\right\rangle$ are governed by the $\Xi K$ mode. The strong decays of both $\left|56,{ }^{4} 10,2,2,5 / 2^{+}\right\rangle$and $\left|70,{ }^{2} 10,2,2,3 / 2^{+}\right\rangle$are governed by the $\Xi(1530) K$ mode. While the $\left|56,{ }^{4} 10,2,2,3 / 2^{+}\right\rangle$and $\left|70,{ }^{2} 10,2,2,3 / 2^{+}\right\rangle$states mainly decay into $\Xi K$ and $\Xi(1530) K$ channels.

It should be mentioned that the $\Omega(2250)$ resonance with a width of $\Gamma=55 \pm 18 \mathrm{MeV}$ listed in PDG [1] may be a good candidate for $\left|56,{ }^{4} 10,2,2,5 / 2^{+}\right\rangle$. The $\Omega(2250)$ was seen in the $\Xi(1530) K$ and $\Xi^{-} \pi^{+} K^{-}$channels. The measured mass of $\Omega(2250)$ is consistent with the quark model predictions [4,8]. Assigning it as $\Omega(2250)$, the total width is predicted to be

$$
\Gamma_{\text {total }}^{\text {th }}[\Omega(2250)]=29 \mathrm{MeV} \text {. }
$$

Its strong decays are dominated by the $\Xi(1530) K$ mode, while the decay rate into the $\Xi K$ is sizeable. The partial width ratio between $\Xi(1530) K$ and $\Xi K$ is predicted to be

$$
\frac{\mathcal{B}[\Omega(2250) \rightarrow \Xi(1530) K]}{\mathcal{B}[\Omega(2250) \rightarrow \Xi K]} \simeq 6.1
$$

Both the decay width and decay mode are consistent with the observations.
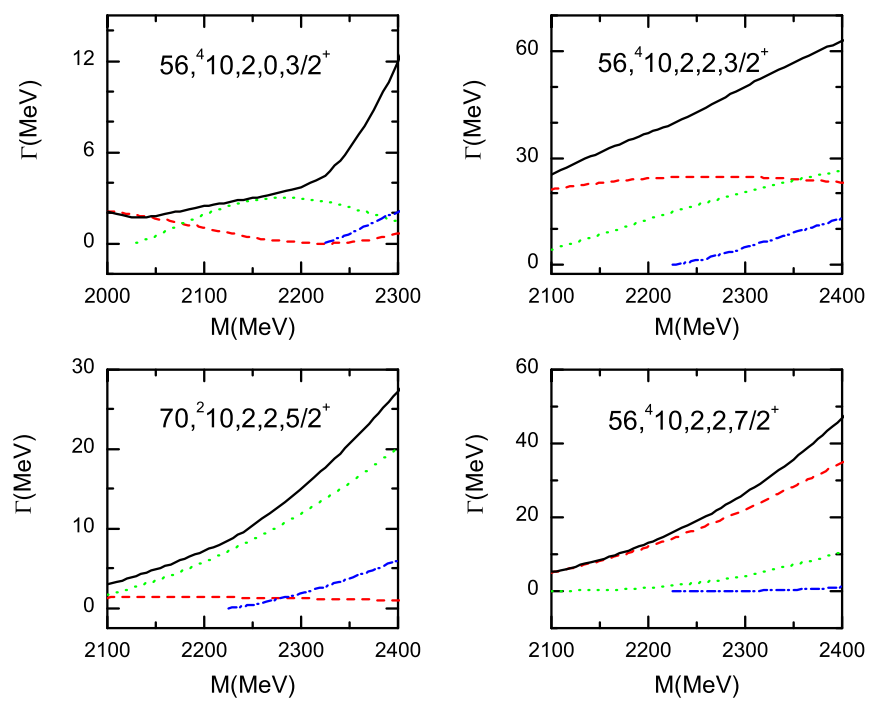

FIG. 2. The strong decay properties of the $2 S$ - and $1 D$-wave states in the $N=2$ shell. 

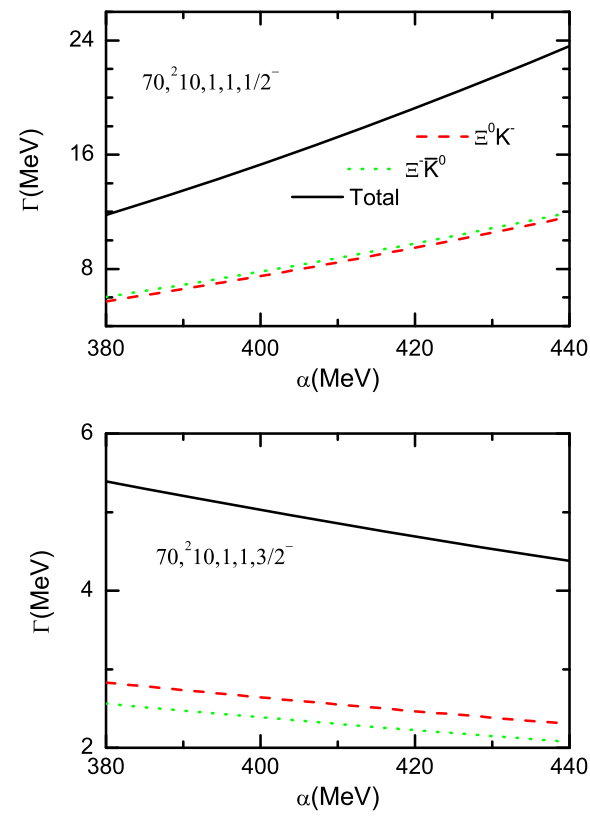

FIG. 3. The strong decay properties of the $1 P$-wave states in the $N=1$ shell as a function of the parameter $\alpha$ with a mass of $M=2012 \mathrm{MeV}$.

As a whole, the $1 D$ wave states are relatively narrow states with a typical width of $10 \mathrm{~s} \mathrm{MeV}$. They mainly decay into $\Xi K$ and/or $\Xi(1530) K$ final states. To establish these missing $1 D$ wave states, observations in both the $\Xi K$ and $\Xi(1530) K$ channels are expected to be carried out in future experiments.

\section{Effect of $\alpha$}

We have calculated the decay properties of the $\Omega$ states up to the $N=2$ shell. Our results are obtained with the harmonic oscillator wave function, which is only an approximation of the genuine wave function. The only parameter $\alpha$ for the harmonic oscillator wave functions is usually adopted the standard value $\alpha \simeq 400 \mathrm{MeV}$. However, the parameter $\alpha$ still bares some uncertainties, which may affect our conclusions.

To see the sensitivities of our results to the uncertainties of the parameter $\alpha$, as an example, we plot the decay widths of the two $1 P$ wave states $\left|70,{ }^{2} 10,1,1,1 / 2^{-}\right\rangle$and $\left|70,{ }^{2} 10,1,1,3 / 2^{-}\right\rangle$as functions of the parameter $\alpha$ in Fig. 3. It is shown that the decay widths for the $1 P$ wave with spin-parity $J^{P}=3 / 2^{-}$are insensitive to the parameter $\alpha$, and the total decay width is $\Gamma \simeq 5 \mathrm{MeV}$ with $\alpha$ within the range of (380-440) MeV. Although the width of $\left|70,{ }^{2} 10,1,1,1 / 2^{-}\right\rangle$shows some sensitivities to the parameter $\alpha$, the width of $\left|70,{ }^{2} 10,1,1,1 / 2^{-}\right\rangle$is always larger than that of $\left|70,{ }^{2} 10,1,1,3 / 2^{-}\right\rangle$. Our conclusion that $\Omega(2012)$ more favors the $\left|70,{ }^{2} 10,1,1,3 / 2^{-}\right\rangle$assignment is not changed by the uncertainties of the parameter $\alpha$. For the $1 D$ and $2 S$ states, we do not show the sensitivities of their decay widths to $\alpha$ here. We also find that although the decay properties depend on the parameter $\alpha$ more or less, the uncertainties of $\alpha$ cannot change our main conclusions.

\section{SUMMARY}

In the present work, we carry out a systematic study of the OZI allowed two-body strong decays of $\Omega$ resonances up to the $N=2$ shell within the chiral quark model. For the newly observed $\Omega(2012)$ state, we give a possible interpretation in theory. Meanwhile, we give the predictions for the decay properties of the $1 D$ wave states and hope to provide helpful information for searching these missing $\Omega$ states in the future.

The newly observed $\Omega(2012)$ state is most likely to be explained as the $1 P$ wave state with $J^{P}=3 / 2^{-}$, $\left|70,{ }^{2} 10,1,1,3 / 2^{-}\right\rangle$. Meanwhile, with the present information from experiments, we cannot completely rule out the $\Omega(2012)$ as the assignments of the $1 P$ wave state $\left|70,{ }^{2} 10,1,1,1 / 2^{-}\right\rangle$with $J^{P}=1 / 2^{-}$and the $2 S$ wave state $\left|56,{ }^{4} 10,2,0,3 / 2^{+}\right\rangle$with $J^{P}=3 / 2^{+}$.

The $\Omega(2250)$ resonance listed in PDG may be a good candidate for the $J^{P}=5 / 2^{+} 1 D$ wave state $\left|56,{ }^{4} 10,2,2,5 / 2^{+}\right\rangle$. Generally, the $1 D$ wave states are relatively narrow states with a typical width of $10 \mathrm{~s} \mathrm{MeV}$. They mainly decay into $\Xi K$ and/or $\Xi(1530) K$ final states. To establish these missing $1 D$ wave states, observations in both the $\Xi K$ and $\Xi(1530) K$ channels are expected to be carried out in future experiments.

\section{ACKNOWLEDGMENTS}

We would like to thank Shi-Lin Zhu for very helpful discussions. This work is supported by the National Natural Science Foundation of China under Grants No. 11775078. This work is also, in part, supported by China Postdoctoral Science Foundation under Grant No. 2017M620492.
[1] C. Patrignani et al. (Particle Data Group), Review of particle physics, Chin. Phys. C 40, 100001 (2016).

[2] J. Yelton et al. (Belle Collaboration), Observation of an excited $\Omega^{-}$baryon, arXiv:1805.09384.
[3] Y. Oh, $\Xi$ and $\Omega$ baryons in the Skyrme model, Phys. Rev. D 75, 074002 (2007).

[4] S. Capstick and N. Isgur, Baryons in a relativized quark model with chromodynamics, Phys. Rev. D 34, 2809 (1986). 
[5] R. N. Faustov and V. O. Galkin, Strange baryon spectroscopy in the relativistic quark model, Phys. Rev. D 92, 054005 (2015).

[6] U. Loring, B. C. Metsch, and H. R. Petry, The Light baryon spectrum in a relativistic quark model with instanton induced quark forces: The Strange baryon spectrum, Eur. Phys. J. A 10, 447 (2001).

[7] J. Liu, R. D. McKeown, and M. J. Ramsey-Musolf, Global analysis of nucleon strange form factors at low $Q^{2}$, Phys. Rev. C 76, 025202 (2007).

[8] K. T. Chao, N. Isgur, and G. Karl, Strangeness -2 and -3 baryons in a quark model with chromodynamics, Phys. Rev. D 23, 155 (1981).

[9] Y. Chen and B. Q. Ma, Light flavor baryon spectrum with higher order hyperfine interactions, Nucl. Phys. A831, 1 (2009).

[10] C. S. An, B. C. Metsch, and B. S. Zou, Mixing of the lowlying three- and five-quark $\Omega$ states with negative parity, Phys. Rev. C 87, 065207 (2013).

[11] C. S. Kalman, $P$ wave baryons in a consistent quark model with hyperfine interactions, Phys. Rev. D 26, 2326 (1982).

[12] M. Pervin and W. Roberts, Strangeness -2 and -3 baryons in a constituent quark model, Phys. Rev. C 77, 025202 (2008).

[13] C.S. An and B.S. Zou, Low-lying $\Omega$ states with negative parity in an extended quark model with Nambu-JonaLasinio interaction, Phys. Rev. C 89, 055209 (2014).

[14] G. P. Engel, C. B. Lang, D. Mohler, and A. Schäfer (BGR Collaboration), QCD with two light dynamical chirally improved quarks: Baryons, Phys. Rev. D 87, 074504 (2013).

[15] J. Liang, W. Sun, Y. Chen, W.-F. Qiu, M. Gong, C. Liu, Y.-B. Liu, Z.-F. Liu, J.-P. Ma, and J.-B. Zhang (CLQCD Collaboration), Spectrum and Bethe-Salpeter amplitudes of $\Omega$ baryons from lattice QCD, Chin. Phys. C 40, 041001 (2016).

[16] C. E. Carlson and C. D. Carone, Predictions for decays of radially excited baryons, Phys. Lett. B 484, 260 (2000).

[17] J. L. Goity, C. Schat, and N. N. Scoccola, Analysis of the $\left[56,2^{+}\right]$baryon masses in the $1 / N(c)$ expansion, Phys. Lett. B 564, 83 (2003).

[18] C. L. Schat, J. L. Goity, and N. N. Scoccola, Masses of the 70- Baryons in Large $N_{c}$ QCD, Phys. Rev. Lett. 88, 102002 (2002).

[19] N. Matagne and F. Stancu, Masses of $\left[70, l^{+}\right]$Baryons in the $1 / N_{c}$ Expansion, Phys. Rev. D 74, 034014 (2006).

[20] R. Bijker, F. Iachello, and A. Leviatan, Algebraic models of hadron structure. 2. Strange baryons, Ann. Phys. (N.Y.) 284, 89 (2000).

[21] T. M. Aliev, K. Azizi, and H. Sundu, Radial excitations of the decuplet baryons, Eur. Phys. J. C 77, 222 (2017).

[22] I. M. Narodetskii and M. A. Trusov, Magnetic moments of negative parity baryons from effective hamiltonian approach to QCD, JETP Lett. 99, 57 (2014).

[23] E. Kaxiras, E. J. Moniz, and M. Soyeur, Hyperon radiative decay, Phys. Rev. D 32, 695 (1985).

[24] A. Manohar and H. Georgi, Chiral quarks and the nonrelativistic quark model, Nucl. Phys. B234, 189 (1984).

[25] X. H. Zhong and Q. Zhao, Strong decays of heavy-light mesons in a chiral quark model, Phys. Rev. D 78, 014029 (2008).
[26] X.H. Zhong, Strong decays of the newly observed $D(2550), D(2600), D(2750)$, and $D(2760)$, Phys. Rev. D 82, 114014 (2010).

[27] X. H. Zhong and Q. Zhao, Strong decays of newly observed $D_{s J}$ states in a constituent quark model with effective Lagrangians, Phys. Rev. D 81, 014031 (2010).

[28] L. Y. Xiao and X. H. Zhong, Strong decays of higher excited heavy-light mesons in a chiral quark model, Phys. Rev. D 90, 074029 (2014).

[29] X.H. Zhong and Q. Zhao, Charmed baryon strong decays in a chiral quark model, Phys. Rev. D 77, 074008 (2008).

[30] L. H. Liu, L. Y. Xiao, and X. H. Zhong, Charm-strange baryon strong decays in a chiral quark model, Phys. Rev. D 86, 034024 (2012).

[31] L. Y. Xiao and X. H. Zhong, $\Xi$ baryon strong decays in a chiral quark model, Phys. Rev. D 87, 094002 (2013).

[32] H. Nagahiro, S. Yasui, A. Hosaka, M. Oka, and H. Noumi, Structure of charmed baryons studied by pionic decays, Phys. Rev. D 95, 014023 (2017).

[33] K. L. Wang, L. Y. Xiao, X. H. Zhong, and Q. Zhao, Understanding the newly observed $\Omega_{c}$ states through their decays, Phys. Rev. D 95, 116010 (2017).

[34] K. L. Wang, Y.X. Yao, X. H. Zhong, and Q. Zhao, Strong and radiative decays of the low-lying $S$ - and $P$-wave singly heavy baryons, Phys. Rev. D 96, 116016 (2017).

[35] L. Y. Xiao, K. L. Wang, Q.f. Lu, X. H. Zhong, and S. L. Zhu, Strong and radiative decays of the doubly charmed baryons, Phys. Rev. D 96, 094005 (2017).

[36] Y.X. Yao, K. L. Wang, and X. H. Zhong, Strong and radiative decays of the low-lying $D$-wave singly heavy baryons, arXiv:1803.00364.

[37] Q. Zhao, J. S. Al-Khalili, Z. P. Li, and R. L. Workman, Pion photoproduction on the nucleon in the quark model, Phys. Rev. C 65, 065204 (2002).

[38] Z. P. Li, The Threshold pion photoproduction of nucleons in the chiral quark model, Phys. Rev. D 50, 5639 (1994).

[39] Z. P. Li, H. X. Ye, and M. H. Lu, An Unified approach to pseudoscalar meson photoproductions off nucleons in the quark model, Phys. Rev. C 56, 1099 (1997).

[40] B. Sengl, Ph. D. thesis, University of Graz, Graz, 2006.

[41] Z. P. Li, The Kaon photoproduction of nucleons in the chiral quark model, Phys. Rev. C 52, 1648 (1995).

[42] Q. Zhao, Z. P. Li, and C. Bennhold, Vector meson photoproduction with an effective Lagrangian in the quark model, Phys. Rev. C 58, 2393 (1998).

[43] X. H. Zhong, Q. Zhao, J. He, and B. Saghai, Study of $\pi^{-} p \rightarrow \eta n$ at low energies in a chiral constituent quark model, Phys. Rev. C 76, 065205 (2007).

[44] T. Melde, W. Plessas, and B. Sengl, Quark-model identification of baryon ground and resonant states, Phys. Rev. D 77, 114002 (2008).

[45] M. V. Polyakov, H. D. Son, B. D. Sun, and A. Tandogan, $\Omega(2012)$ through the looking glass of flavour $\mathrm{SU}(3)$, arXiv: 1806.04427.

[46] T. P. Vrana, S. A. Dytman, and T. S. H. Lee, Baryon resonance extraction from $\pi N$ data using a unitary multichannel model, Phys. Rep. 328, 181 (2000). 